\title{
Le dialogue simonien : enjeux, formes, déviances
}

\author{
Marie-Albane Rioux-Watine
}

\section{(2) OpenEdition}

Journals

Édition électronique

URL : http://journals.openedition.org/ccs/567

DOI : $10.4000 /$ ccs.567

ISSN : 2558-782X

\section{Éditeur :}

Presses universitaires de Rennes, Association des lecteurs de Claude Simon

\section{Édition imprimée}

Date de publication : 30 avril 2007

Pagination : 23-41

ISBN : 9782354120122

ISSN : $1774-9425$

\section{Référence électronique}

Marie-Albane Rioux-Watine, "Le dialogue simonien : enjeux, formes, déviances », Cahiers Claude Simon [En ligne], 3 | 2007, mis en ligne le 20 septembre 2017, consulté le 19 avril 2019. URL : http:// journals.openedition.org/ccs/567; DOI : 10.4000/ccs.567 


\title{
Le dialogue simonien : enjeux, formes, déviances
}

\author{
Marie-Albane RIOUX-WATINE
}

«Je n'ai pas le don de parole», affirme S., le personnage écrivain du Jardin des Plantes (JP, 957), alter ego de Simon. Il reprend d'ailleurs mot pour mot une déclaration de Simon lui-même, datant de 1971 : «Pardonnez-moi : je n'ai pas le don de parole (c'est d'ailleurs probablement la raison pour laquelle j'écris : si tu veux être peintre, disait Matisse, commence par te couper la langue !) ${ }^{1}{ }^{1}$ 'écrivain se définit ainsi comme homme à la langue coupée, condamné au silence par une écriture qui, par définition, amuït toute voix. Et il faut bien reconnaître qu'au milieu de ses amis nouveaux romanciers, Simon ne se fait pas remarquer par son verbe. Alors que les uns enregistrent des phonotextes et que les autres parlent à la radio ou à la télévision, Simon reste obstinément fidèle à sa politique de silence. Il prétend que la voix n'entre pour rien dans l'écriture, considère ses romans comme illisibles à haute voix ${ }^{2}$ et affirme ne jamais passer ses textes au gueuloir ${ }^{3}$.

Pourtant, la voix des personnages occupe bien une place cen-

1 «Le métier de romancier», lettre de Claude Simon adressée au Monde en 1971, publiée le 19 octobre 1985.

2 C. Simon, "Claude Simon, à la question », Claude Simon, analyse, théorie, colloque de Cerisy, UGE, coll. 10/18, 1975, p. 215.

3 Ibid., p. 214. 
traie dans les textes simoniens. L'œuvre n'est pas avare en longs passages de description des voix des personnages, et il faut d'autre part reconnaitre l'effet particulier produit par les dialogues simoniens, leur grand effet de réel, et l'effort de mimesis de voix vive qu'ils supposent.

C'est cette ambiguïté fondamentale de la voix chez Simon, à la fois rejetée et intégrée, que nous nous proposons de cerner dans cette étude du dialogue simonien. La voix dans l'écriture - et dans celle de Simon en particulier - est un champ d'analyse vaste et protéiforme, parfois insaisissable si l'on tente de l'envisager dans la variété de ses manifestations, qui s'étendent du style parlé à la caractérisation des voix en passant par le rythme. On restreindra cette étude au seul problème du dialogue, qui nous semble explorer les différents chemins d'une paradoxale inscription de la voix vive dans le texte écrit. On partira d'une définition minimale du dialogue, empruntée à S. Durrer, comme « échange de répliques entre deux ou plusieurs personnages $»^{1}$, sans préjuger du type de discours rapporté qui est employé (discours direct, indirect, ou leurs deux variantes libres).

\section{Approche quantitative : l'ambiguïté de l'inscription}

Commençons par quelques constatations quantitatives qui, déjà, mettent au jour une instable politique du dialogue. On a souvent considéré Simon comme peu porté sur les dialogues, peut-être en raison de la mise en page souvent adoptée pour certains d'entre eux, qui renoncent au blanc typographique et ressemblent dès lors au texte continu du récit. Mais l'approche quantitative permet de s'assurer que Simon est bien un écrivain dialogal : dans Fa Route des Flandres, le rapport du nombre de lignes de dialogue sur la totalité du roman s'élève à $33 \%$, ce qui place le roman très loin au-dessus, par exemple, du Rouge et le Noir (22\%), ou de L'Éducation sentimentale $(20 \%)^{2}$. Mais il est vrai qu'avec Fa Route des Flandres, il s'agit d'un cas extrême. La place des dialogues est soumise à de notables variations

\footnotetext{
1 Le Dialogue dans le roman, Nathan Université, « $128 »$, 1999, p. 6.

2 Chiffres cités par S. Diirrer, op. cit.
} 
dans l'ensemble de l'œuvre. Elle est importante dans les romans jusqu'à Histoire, mais ceux de la période formaliste, comme Leçon de Choses et Triptyque, ne comportent que quelques lignes de discours direct, qui ne représentent que $0,6 \%$ de l'ensemble, même si le style parlé est loin d'être absent de Leçon de choses ${ }^{1}$. Dans Les Géorgiques, le dialogue oral, remplacé par l'échange de lettres, est aussi presque absent. Et, bien que la description de paroles soit omniprésente dans L'Invitation, la citation effective de paroles ne représente que $2 \%$ du texte, et encore s'agit-il toujours de répliques solitaires, et donc pas de dialogue au sens strict. Celui-ci fait sa réapparition dans L'Acacia, entre le jeune voyageur et son compagnon mexicain. C'est avec $\mathrm{Le}$ Jardin des Plantes qu'il refait réellement son entrée au cœur du récit (avec un rapport de 15\%). Mais il disparait de nouveau presque complètement dans Le Tramway, texte placé dès ses premières lignes sous l'empire du silence (" Défense de parler au wattman» [Tram., 12]). Le dernier texte de Simon consacre une réelle éviction du dialogue. Le discours y est très rare sous toutes ses formes; le récit prend place dans des lieux structurellement silencieux où personne ne se parle : la convalescence à l'hôpital avec le vieillard (" nous avions tous deux, comme par un accord tacite sur l'inutilité (ou la futilité) de toute parole, gardé le silence » [Tram., 67] $]^{2}$ ), une grande partie de la vie familiale, la pêche à la sardine ${ }^{3}$, la Plage Mondaine vue de loin dans une « totale absence de bruit » (43)...

En résumé, après une période dialogale qui s'étend des premiers

\footnotetext{
Rappelons que selon notre définition restrictive du dialogue, qui suppose au moins une interaction verbale, on ne peut considérer les deux « Divertissements » de Leçon de choses comme des dialogues, même si ces longs monologues impriment de toute évidence une marque fortement oralisée au roman...

2 Voir aussi, p. 117-118, le passage de la jeune femme dans son appareillage, avec la figure dérivative autour du " silence" : les malades "suivaient en silence le passage silencieux de l'impressionnante machine montée sur ses silencieuses roulettes de caoutchouc, guidée par les deux silencieux infirmiers chaussés de sandales $»$.

3 Voir, p. 56, « la longue houle qui mettait longtemps à retomber - et le silence, et seulement, parfois, le frais ruissellement, ici ou là, d'une de ces rides dont la crête se brisait, les lueurs du couchant allumant des reflets de bronze sur l'eau non plus bleue mais d'un vert bouteille allant s'assombrissant, noir à la fin dans le silence noir ».
} 
romans à Histoire, les textes se détournent en partie de l'inscription de la vocalité à partir de la période formaliste, avec toutefois une résurgence dans l'avant-dernier roman, et des phénomènes notables de style parlé hors du cadre strict du dialogue dans Leçon de choses. Plusieurs déterminations fictionnelles et scripturales expliquent en partie cette variation, comme l'absence de personnages constitués dans la période formaliste, ou comme l'invasion du report d'écrit dans Les Géorgiques, qui vient se substituer au report de voix. D'autre part, il est possible que Simon, ayant porté la technique du dialogue à un extrême degré de finesse, se détourne un moment d'une pratique dont il a fait le tour, ne la remobilisant dans La Bataille de Pharsale que pour la mettre à distance : la séquence intitulée " Conversation », relatant un dialogue maladroit entre Charles et la femme du peintre, semble maximaliser tous les «bruits » communicationnels ${ }^{1}$ qui ont coutume, de façon plus ou moins voyante, d'accompagner toute interaction simonienne. A partir de cette distanciation ironique, le dialogue s'efface durablement de l'œuvre ${ }^{2}$. Mais plus profondément, cette inconstance du dialogue est à rapporter à l'ambiguïté de la relation des textes simoniens à la voix, rapport à la fois de rejet (les textes prenant acte du fait que la voix est, par essence, la grande absente de l'écriture) et de fascination. En effet, la voix est exempte des critiques métaphysiques et politiques qui touchent l'écriture, souvent caractérisée comme vecteur d'oppression bureaucratique ou contre-révolutionnaire ${ }^{3}$. La voix, qui autorise la communication

\footnotetext{
1 Charles ne prend conscience des paroles de la femme qu'avec intermittence, elle lui parle à travers un rideau qui semble faire "barrière" aux sons, les deux locuteurs ne cessent de s'interrompre l'un l'autre... Cette «conversation», pourtant formellement très aboutie avec ses diverses formes de discours rapporté et sa typographie minimale, est une interaction ratée, à l'image de la voix du voisin qu'on entend " chantonner" " comme à côté de la mélodie » de la guitare. La voix ici chante son chant du cygne, comme le son de la guitare comparé à " certaines voix d'enfants quelque chose d'à la fois limpide et à la limite de la tension de la rupture » $(B P, 647-648)$..

2 La place charnière de cette conversation de La Bataille de Pharsale dans l'évolution de la pratique simonienne nous a été suggérée par $\mathrm{M}$. Bertrand, que nous remercions ici.

3 Pensons par exemple aux hommes de l'ombre qui reprennent en main Barcelone et font donner la chasse à $\mathrm{O}$. ; ils ne parlent presque jamais, mais tirent leur puissance de décrets tapés à la machine au moyen desquels ils réduisent les « orateurs passionnés » au silence $(\mathrm{G}, 357)$.
} 
en présence, apparaît fugacement comme le medium privilégié de la présence de l'être et du réfèrent ${ }^{1}$.

D'ailleurs, si Simon rejette régulièrement le dialogue au sein de ses romans, il reste que ceux-ci jouent un rôle énonciatif central dans certains d'entre eux. L'importance quantitative est alors renforcée par une place particulière, qui touche à l'origine fictive de l'écriture. Nous ne prendrons qu'un exemple, Le Jardin des Plantes, mais des études similaires peuvent être menées pour L'Acacia, La Route des Flandres ou Le Vent.

Dans Le Jardin des Plantes, l'attitude du narrateur envers sa conversation enregistrée avec un journaliste est constamment ambiguë : tout en laissant transparaître une certaine animosité envers l'instrument qui fixe la parole (on se souvient que S. répugne à être enregistré), le narrateur ne dédaigne pas de nous livrer la teneur de cet entretien - et il n'est dès lors pas interdit de penser que, fictivement du moins, il s'est servi de l'enregistrement pour la retranscription du dialogue que nous lisons. D'autre part, on sait que c'est à la suite d'une question du journaliste que S. se demande comment on pourrait représenter l'ensemble simultané des différentes péripéties de la guerre. Il imagine alors un procédé ressemblant aux films où l'on projette des images différentes sur plusieurs écrans, ce qui « est impossible en parlant ou en écrivant. Mais on peut quand même essayer» (JP, 1055) : c'est donc de l'entretien que provient, à l'intérieur de la fiction, l'idée de la mise en page particulière du début du roman. Le dialogue se donne ici comme point d'origine fictif de l'écriture.

La position originelle de l'entretien se manifeste également au niveau énonciatif. Le dialogue avec le journaliste, qui réapparaît à intervalles réguliers dans le texte, quitte peu à peu son statut de fragment épars parmi d'autres pour prendre une position surplombante. Fugitivement, les fragments concernant la guerre se donnent comme la narration qui est faite de cette expérience au journaliste. À deux reprises dans le roman en effet, les fragments de la guerre sont,

1 Nous renvoyons ici à notre thèse, La voix et la frontière. Sur Claude Simon, Paris, H. Champion, 2007. 
de façon inattendue, subordonnés à l'entretien. Ils s'interrompent brutalement sur une réaction orale du journaliste :

[...] dans ces hameaux ou ces villages tous semblables aussi du Wurtemberg, du Hanovre ou de la Saxe, toujours désespérément mornes et, dans le fond, le flanc pâle d'un coteau désespérément morne aussi, désespérément gris... - Mais c'est peut-être là l'explication, dit le journaliste. L'explication de quoi ? dit S. L'ennui, dit le journaliste. Simplement ça: l'ennui. (JP, 1037)

[...] le général allemand n'arrivant pas à se faire entendre, s'égosillant, essayant en vain de faire cesser le feu, et quand il y parvint ce fut pour voir de tous les côtés les silhouettes de soldats qui jetaient leurs armes, levant les bras, se rendant, ahuris, mal réveillés, sortant des maisons, des garages, des tentes où ils dormaient, des cours, des hangars, des chemins de traverse encombrés de canons, de mitrailleuses, d'affûts, de chars, de matériel de toute sorte, intact, abandonné (le journaliste s'exclamant: Mais ce n'est pas possible! Vous voulez dire que... qu'ils... qu'on... Mais c'est incroyable ! C'est... Vous... Je veux dire : ils...). (JP, 1052-1053)

Ces fragments concernant la guerre, qui semblent au départ autonomes, se trouvent subitement rattachés à l'entretien par une réplique inattendue du journaliste. Par contamination, on est conduit à envisager que tout ce qui a trait à la guerre est issu du récit qu'en fait $\mathrm{S}$. au journaliste ; ces fragments a priori indépendants seraient en réalité subordonnés à un dialogue originel. Dans le deuxième passage cité, on observe d'ailleurs un mouvement caractéristique quant à l'ambiguïté de la position de la voix dans les textes simoniens : au moment même où le fragment mime la défaillance de la parole, en particulier par l'inintelligibilité des ordres du général et le bégaiement du journaliste, se trouve réaffirmée la position originelle et intégrative de l'entretien dans la construction romanesque.

Ces court-circuitages actantiels inattendus laissent penser qu'une grande partie des fragments tourne autour de ce centre dialogal dont l'enregistrement a gardé trace. D'autres centres originels peuvent être distingués : ainsi les fragments russes sont sans doute dépen- 
dants de la conversation de $\mathrm{S}$. avec sa compagne, dans une salle de bain où il raconte son voyage. Les fragments concernant Novelli, le lecteur le comprend peu à peu, trouvent leur origine dans les confidences que le peintre a faites à S., comme l'indique l'apparition de verbes de discours ou d'incises, toujours inattendues, dans certains fragments qui semblaient indépendants

Arrêté par les Allemands Gastone N... fut envoyé au camp d'extermination de Dachau et torturé Il dit qu'après non seulement il ne pouvait plus supporter la vue d'un Allemand ou d'un uniforme mais même celle d'un être dit civilisé $(J P, 913)$

Le soir il mangèrent tous ensemble les poissons, et d'autres choses (assez répugnantes, dit-il) qu'il se força à avaler. (JP, 1078 ; nous soulignons)

Finalement, une partie du Jardin est ainsi progressivement saisie par le lecteur comme transcription de discours proférés ou entendus par un narrateur mouvant, dans un lieu et un temps indécis. La subordination à la parole, en effet, ne s'avoue pas d'entrée de jeu, et son lieu reste toujours inassigné.

On ne peut développer ici ce point, mais on voit la parenté de cette analyse avec la structure de La Route des Flandres, ou encore avec ce que l'on peut appeler l'effet d'enquête de L'Acacia, qui fait du roman la relation de discours croisés recueillis par un narrateur en quête de son origine. De ce point de vue, l'origine (de l'écriture, de l'existence) est intimement liée à l'oralité et au dialogue, mais cette origine se révèle le plus souvent énonciativement inassignable. Remarquons toutefois que Le Jardin des Plantes porte à son paroxysme la multiplication des voix sans ressaisie globale ; contrairement à ce qui se passe dans L'Acaria, La Foute des Flandres ou Le Lent, la majorité des fragments restent indépendants, échappant au crible d'un narrateur venant après coup ordonner la parole entendue ou tenter, même fugacement, d'en extraire un sens.

2. Les formes du dialogue

C'est selon cette relation complexe à la voix, simultanément rejetée en périphérie et installée au centre de l'écriture, à la fois lieu de 
l'origine et signe de différance (au sens derridien), qu'il faut selon nous se pencher sur les formes diverses que prennent les dialogues dans l'œuvre, en les abordant d'un point de vue énonciatif, typographique et stylistique.

\section{Les formes du discours rapporté (DR)}

D'un point de vue énonciatif et syntaxique, c'est le DR qui permet l'inscription mimétique des voix des personnages. Il suppose une différenciation entre un discours citant et un discours cité ; dans les cas qui nous intéressent, le discours citant est le niveau de l'écriture, le discours cité est celui de la voix, ou du moins de la mimesis de voix des personnages. C'est donc dans le discours rapporté que se rencontrent, fictivement du moins, les deux substances de l'expression, voix et écriture.

On sait qu'on répertorie en général 4 types de discours rapporté, le discours direct libre (DDL), le discours direct (DD), le discours indirect libre (DIL) et le discours indirect (DI). On peut envisager que du DDL jusqu'au DI, il existe un continuum. Le DDL suppose l'entrée en force de la parole vive dans l'écrit ; c'est la parole même de l'autre qui est citée, avec ses inflexions propres (les modalités phrastiques, par exemple, sont conservées) ; seul le changement de repérage énonciatif (repères personnels et temporels) signale le décrochement entre discours citant et cité. À l'autre bout de la chaîne, le DI intègre au contraire la voix en l'assimilant au niveau de l'écriture. Les paroles sont syntaxiquement subordonnées au discours citant, de façon homogène. Il n'y a plus de décrochement dans le repérage énonciatif ; mais simultanément le discours perd ses inflexions propres (les modalités disparaissent). Le DI est souvent davantage perçu comme traduction homogénéisante des paroles que comme citation hétérogène des paroles de l'autre.

Tout écrivain, pour inclure une représentation de la voix dans le texte, se trouve donc confronté à ce dilemme : intégrer la voix sans hiatus, dans le discours indirect, mais taire du même coup ses inflexions propres (la modalité, l'intonation, et même l'idiolecte de l'autre) ; ou citer fidèlement les paroles, dans une volonté de mimesis maximale, mais alors le décrochage énonciatif et souvent typogra- 
phique dénonce ces paroles comme hétérogènes, comme autre du texte, comme inclusion (au sens où l'on parle d'inclusion de matière hétérogène dans une pierre).

Simon prend parti, et opte très majoritairement pour le dialogue au DD. Le DI ou le DIL sont minoritaires dans son écriture : on peut donc parler de volonté d'inclure la voix même de l'autre, avec ses inflexions - mais cela n'a rien de particulièrement remarquable, puisque la solution du DD est la norme, le dialogue au DI restant une pratique rare en littérature, sans doute en raison de sa relative lourdeur syntaxique.

Ce qui en revanche est particulièrement révélateur de ses questionnements propres, ce sont les solutions intermédiaires que Simon découvre dans Le Jardin des Plantes et dans Le Tramway. Dans ces deux romans, on voit apparaître des formes mixtes de discours rapporté qui permettent de jouer avec la nécessaire hétérogénéité énonciative du DR et de la réduire en partie.

\section{Ainsi dans ce passage, extrait du Jardin des Plantes:}

Il a dit Le thème de la guerre revient avec insistance chez vous. On a même avancé que c'est là la clé qui conditionne tout ce que vous avez écrit et que... J'ai dit que Ho c'était tout de même un peu exagéré, un peu réducteur, que j'avais tout de même écrit pas mal d'autres choses et que... Mais il m'a coupé, il a dit Tout de même admettez que... (JP, 955)

Du point de vue typographique, les répliques de $\mathrm{S}$. et celles du journaliste semblent homogènes : verbe de parole introducteur, absence des deux-points introducteurs du DD, majuscule de démarcation. Mais elles comportent une différence de taille : dans la réplique de S. (« j'ai dit que Ho »), les trois «que» subordonnants et le plusque-parfait de concordance indiquent l'intégration de la parole dans le DI... ou du moins dans une apparence de DI. Car l'interjection «Ho» révèle une discordance. Pour tous les linguistes du discours rapporté, l'interjection est par excellence une partie du discours qui ne peut être intégrée telle quelle au DI, car comme le rappelle D. Maingueneau, « il est impossible de mettre au DI un certain nombre 
d'éléments qui figurent au DD : onomatopées, interjections, vocatifs, exclamatifs, énoncés inachevés, en langue étrangère ${ }^{1}$.

Après Ann Banfield ${ }^{2}$, Laurence Rosier parle justement de « dire que + interjection » comme « mariage impossible », et qui peut même être considéré comme un repère stable qui «cristallise la frontière syntaxique entre DD et DI, cette irréductibilité qui fait exister la dichotomie $»^{3}$. Irréductibilité illusoire, puisqu'elle recense aussitôt de nombreux exemples littéraires d'interjections en discours indirect, et pas seulement au $\mathrm{XX}^{\mathrm{e}}$ siècle, puisque la tournure remonte au moins à Rabelais.

L'interjection fait partie de ce qu'elle appelle les « discordantiels d'énonciation", définis comme "tous les mots ou locutions permettant d'attirer le dire du narrateur (rapporteur) vers le dit du personnage [...] : ils confrontent le discours citant au discours cité $»^{4}$. Ils autorisent donc, dans le cadre qui nous occupe, un contact plus proche de la voix et de l'écrit. Ici, la voix est intégrée syntaxiquement; elle est donc homogène énonciativement, mais elle garde malgré tout, grâce à l'interjection, certaines de ses inflexions propre. Le dilemme du discours rapporté, que nous évoquions plus haut, se résout dans un moyen terme, une solution hybride qui ménage à la fois l'inscription de la voix au cœur même du récit, sans hiatus énonciatif, et l'inflexion de la parole propre. Plus loin dans le texte, les formes du DR sont plus variées encore

J'ai dit que Oui c'était vrai que ça revenait souvent mais... Il a dit Pourquoi ? J'ai dit que Vous savez je n'ai pas beaucoup d'imagination alors à part mes tout premiers bouquins qui n'étaient pas très fameux les suivants ç'a toujours été plus ou moins à partir de choses que j'ai vécues, de mes expé-

1 Éléments de linguistique pour le texte littéraire, Bordas, 1986, p. 86.

2 Dans Phrases sans parole. Théorie du récit et du style indirect libre, Seuil, 1995 [trad. fr. de Unspeakable Sentences. Narration and Representation in the Language of Fiction, London, Routledge and Kegan Paul, 1982], la linguiste générativiste recense exhaustivement l'ensemble des constructions qui sont inacceptables au discours indirect, p. 61-70.

3 Le discours rapporté. Histoire, théories, pratiques, Duculot, "Champs linguistiques », 1999, p. 225.

4 Ibid., p. 152. 
riences personnelles, ou encore de vieux papiers de famille, tout ça... Il a dit Oui qu'il savait mais que ce qui l'intéressait c'était ça : mon expérience personnelle, alors j'ai dit que Ma foi il se trouvait qu'entre autres il y avait eu la guerre [...]. $(J P, 955)$

Nous retrouvons tout d'abord une forme de DI + discordantiel d'énonciation (la prophrase "oui », moins déviante toutefois que l'interjection). La troisième réplique, elle, relève de ce que l'on est bien obligé d'appeler un DD avec marque de subordination: elle garde toutes les caractéristiques du DD, mais elle comporte un " que » subordonnant et une majuscule démarcative. La réplique suivante accumule sans hiatus, dans une sorte de zeugme syntaxique, une structure de DD ( Il a dit Oui ») et une structure de DI (« il a dit qu'il savait $»)$.

Dans Le Tramway figure un véritable cas d'école. La forme du DR devient réellement indécidable :

[...] elle m'a répondu sur un ton de soudaine indignation que Monsieur, on avait bien essayé d'en pourvoir ces toilettes mais qu'alors les malades oui Monsieur savez-vous ce qu'ils y avaient fait, eh bien ils y avaient fait caca! (Tram., 111)

Les indices indéniables de DI (maintien du «que » subordonnant, plus-que-parfait de concordance) côtoient de non moins certaines preuves de DD (appellatif « Monsieur », interjection « eh bien », présent d'énonciation, maintien de la modalité exclamative ponctuée, maintien de l'inversion interrogative, interrogation oratoire à valeur thématisante, maintien du « vocable enfantin et cru » (« caca») propre à l'énonciateur cité)...

On voit ici l'extrême labilité du DR dans les derniers romans de Simon. Ces pratiques présentent l'intérêt de gommer les frontières qui séparent le niveau de l'écriture du niveau de la voix, en permettant à la fois leur inclusion homogénéisante et le maintien de l'inflexion propre. Dans ces formes mixtes se dit la recherche d'un texte qui serait traversé, sans hiatus ni couture, par son autre absolu, la voix. 
Aspect prosodique et typographique : les formes du dialogue

La même tension vers l'effacement de la frontière écrit/voix est à l'œuvre au niveau de la typographie des dialogues. La pratique simonienne, de ce point de vue, a considérablement varié ; il est possible de mettre au jour une typologie des dialogues, ainsi qu'une chronologie de leur création. Cette intense créativité est à rapporter à tout un contexte critique : on sait que le dialogue, dès les débuts du Nouveau Roman, se trouve sur la sellette. Le dialogue classique et ses incises répétitives deviennent la cible des essayistes, et en particulier de Sarraute, dont on peut convoquer pour mémoire un célèbre passage de «Conversation et sous-conversation », datant de 1956 :

Dès lors, rien n'est moins justifié que ces grand alinéas, ces tirets par lesquels on a coutume de séparer brutalement le dialogue de ce qui le précède. Même les deux points et les guillemets sont encore trop apparents, et l'on comprend que certains romanciers (Joye Cary notamment) s'efforcent de fondre, dans la mesure du possible, le dialogue avec son contexte en marquant simplement la séparation par une virgule suivie d'une majuscule. Mais plus gênants encore et plus difficilement défendables que les alinéas, les tirets, les deux points et les guillemets, sont les monotones et gauches : dit Jeanne, répondit Paul, qui parsèment habituellement le dialogue ; ils deviennent de plus en plus pour les romanciers actuels ce qu'étaient pour les peintres, juste avant le cubisme, les règles de la perspective : non plus une nécessité, mais une encombrante convention. ${ }^{1}$

On voit que ce qui est en jeu ici, c'est la « séparation » abusive du dialogue d'avec le récit. Il faut "fondre » ces énonciations hétérogènes pour passer insensiblement de l'un à l'autre, et cette fusion s'accomplira par l'allégement du marquage typographique. Il est évident que cette réflexion, tout autant que l'influence de Faulkner, a fortement marqué l'écriture simonienne dans les quelques années qui suivent. 
C'est dans Le Vent, L'Herbe et La Route des Flandres que se concentre toute la créativité simonienne en matière de dialogue. Si l'on prend en compte différents critères comme la forme du discours attributif (incise, type « et moi »/« et lui », ou absence), la présence ou non des deux-points et des guillemets, de la majuscule démarcative en début de réplique, et le retour à la ligne entre les répliques, 13 types différents sont expérimentés entre Le Vent et La Route des Flandres. Dans les textes suivants, seuls trois nouveaux types apparaîtront, et la variété de types utilisés au sein d'un même roman ne dépassera pas 3 ou 4 occurrences différentes.

Or, cette intense créativité va régulièrement, dans ces années, dans le sens d'un allègement du marquage. Dans L'Herbe apparaît un type qui porte à son paroxysme la disparition de la démarcation, sans discours attributif, sans tiret ni guillemet sauf en début et en fin de dialogue, sans retour à la ligne, avec simplement une virgule et une majuscule démarcative entre chaque réplique :

[...] les deux voix se répondant, alternant, se mariant, se fondant dans sa mémoire en une sorte de bloc unique, indivisible, questions et réponses soudées dans cette espèce d'implacable et absurde enchaînement de tout dialogue, de toute parole : «Qu'est-ce que tu as, Rien, Si qu'est-ce que tu as, Rien je te dis que veux-tu que j'aie qu'est-ce qui te prend maintenant qu'est-ce, Pourquoi fais-tu cette tête, Quelle tête, Cette tête, Je ne fais aucune, Non $\mathrm{m}$ crois peut-être que je suis idiot tu te figures que je ne sais pas très bien quand tu as quelque chose même quand tu essaies [...]. (Herbe, 155)

Ce type extrême d'effacement reçoit une justification dans le cotexte : la «fusion", le "mariage» des voix, incongru dans cette scène de divorce émotionnel, sont tributaires du travail de la mémoire, qui a comme toujours tendance à tout mettre sur le même plan. Il n'empêche qu'indépendamment de ce contexte narratif accidentel, la tendance à l'allègement des démarcations typographiques subsiste, et Simon s'éloigne définitivement des conventions classiques qu'il respectait scrupuleusement avant Le Vent.

Dans les trois derniers romans dominent les types qui renoncent au marqueur le plus visible, le plus associé visuellement au dialo- 
gue, c'est-à-dire au retour à la ligne inter-répliques. Le blanc limitatif s'efface : la voix représentée semble trouver sa place au cœur de la narration écrite, la frontière voix/écriture est comme traversée, et le texte ne marque plus la suture, le bord différentiel. Mais alors, simultanément, cette intrusion de la voix des personnages au cœur de l'écriture rend la voix narrative moins uniforme, moins autonome. Se dessine alors peut-être, loin d'un sujet classique source et maître de sa parole, intériorité nettement séparée du monde et de la rumeur environnante, un sujet d'énonciation plus moderne, traversé des dires externes, "parlé plutôt qu'il ne parle », comme l'ont décrit diverses approches théoriques (pluriliguisme bakhtinien ou psychanalyse lacanienne). La narration mémorielle est aussi une épreuve d'altérisation, où la frontière de la voix propre avec les voix autres se trouve en partie dissoute. Pour reprendre allusivement les analyses de J. Authier-Revuz sur l'hétérogénéité constitutive et l'hétérogénéité montrée ${ }^{1}$, ces textes, en gommant l'indexation des voix autres, proposent une négociation plus risquée avec l'altérité constitutive qui traverse tout sujet.

\section{Le style parlé}

C'est cette même tendance à la déconstitution subjective dans le travail de la voix que met en évidence l'étude du style parlé simonien dans les dialogues. On sait que la refonte plus ou moins artificielle de la langue parlée dans le texte littéraire fait partie des préoccupations centrales de l'époque ${ }^{2}$. Il est aisé de repérer chez Simon les marques propres à cet artefact littéraire ${ }^{3}$ qu'est le style parlé. On peut évoquer entre autres les marques phoniques qui, par aphérèse ou apocope, marquent graphiquement une prononciation courante,

1 Voir en particulier « Hétérogénéité montrée et hétérogénéité constitutive : éléments pour une approche de l'autre dans le discours », DRLAV, 26, p. 91-151, 1982 et « Hétérogénéités énonciatives », Langages, 73, p. 98-111.

2 Nous renvoyons à ce sujet à l'étude de J.-P. Martin, La bande sonore : Beckett, Céline, Duras, Genet, Perec, Queneau, Sarraute, Sartre, Paris, Corti, 1998.

3 S. Durrer rappelle à juste titre que « [1]a langue parlée envisagée par les romanciers ne correspond pas à la "réalité" ; il s'agit d'un artefact, c'est-à-dire d'une sélection de traits qui connotent l'oralité. », op. p. 25. 
et qui est toujours connotée socialement. Ce passage de Fa Rouie des Flandres, où seul Wack la pratique, est révélateur de ce point de vue :

Wack $[\ldots]$ disant à la fin de mauvaise grâce, furieux : «Je l'sais !» [...], et Blum : «Et où est le mari ? En balade à la ville ? », et Wack se retournant d'une pièce, disant : « En balade comme toi, spèce de con : 'vec un casque sur la tête !», et Blum : «T'as oublié de m'appeler sale youpin. » (RF, 236)

On relève aussi des marques lexicales, qui consistent là encore à employer des lexies de registre familier pour connoter l'origine sociale. Diverses "fautes" syntaxiques apparaissent, avec la même visée - on sait que traditionnellement, la langue parlée est considérée comme fautive, et divers barbarismes syntaxiques permettent aussi de connoter, un peu artificiellement peut-être, le style parlé. On relève ainsi fréquemment des omissions $d u$ «ne » discordanciel de négation: « je sais pas ce que je donnerais » $(R F, 237)$, «j'ai pas vu d'église ", "y a pas d'autre moyen de l'empêcher de trottiner» (238), "c'est pas marrant pour lui », "j'aurais jamais cru» (Hist., 127), «mais oui t'en fais pas », ou encore des relatifs fautifs. Tous ces procédés tendent à recréer à réception une illusion de voix vive venue habiter le texte - mais ils sont assez connus en littérature, et au demeurant assez circonscrits chez Simon, pour que l'on ne s'y attarde pas.

En revanche, certaines particularités syntaxiques semblent plus spécifiquement simoniennes. Il s'agit en particulier de l'intégration régulière, dans le dialogue, de deux constructions qui sont vraiment mimétiques des programmes syntaxiques propres à l'oral, à savoir l'interruption et la reformulation. On sait à quel point l'interruption-aposiopèse est constante dans les dialogues simoniens. Les personnages s'interrompent eux-mêmes au milieu d'une phrase, ou sont interrompus par d'autres, dans un souci de fidélité particulièrement mimétique des interactions verbales réelles. La reprise-écho interrompue participe souvent à cet effet de réel, comme dans ces deux extraits

« mais il en a eu assez il l'a chassée Ou plutôt répudiée ré... Comment dis-tu» $(\mathrm{RF}, 282)$ 
«Mais ils ont tout de même renvoyé la bonne?

- S'ils ont... Sans blague ? Vous n'auriez tout de même pas voulu qu'ils lui donnent une augmentation ?» $(V, 91)$

Plus intéressants encore sont les nombreux cas de corrections-reformulations, cas étendu d'éparnorthose :

- Qu'il dés vous voulez dire qu'uniquement parce que vous pensez que monsieur que votre $\mathrm{p}$ enfin que c'est eu égard au fait qui mais parfaitement parfaitement c'est c'est $(V, 15)$

On peut aborder ces schèmes de reformulation, dans le dialogue, en référence aux cadres de la praxématique. Selon J.-M. Barbéris et B. Maurer ${ }^{1}$, ces « ratages » sont propres au discours oral, et ils s'expliquent par la complexité des opérations cognitives simultanément mises en jeu à l'oral. Dans la construction d'un énoncé oral, trois niveaux temporels se superposent: temps de l'à-dire (où le sujet élabore la programmation du message), temps du dire (temps de l'élocution physique), et temps du dit (mise en mémoire des programmes réalisés antérieurement). Cette juxtaposition de processus cognitifs divers justifie en partie ces inévitables ratages, qui connotent très efficacement la réalité d'une interaction effective. De surcroît - et c'est ce qui nous intéresse ici au premier chef -, ces structures manifestent un sujet en perte de contrôle sur son propre dire, qui se laisse facilement envahir par la parole d'autrui. La déprise sur le monde, dont sont victimes la plupart des personnages simoniens, est aussi une déprise de la voix subjective. Avec ces programmes syntaxiques particuliers, la praxématique parle d'ailleurs d'une « textualité en idem », qui manifesterait une subjectivité floue et mal dégagée de l'altérité.

\section{Déviances}

Il semble pour finir que certaines déviances du dialogue aillent également dans ce sens d'une relative dissolution des frontières de

\footnotetext{
1 «Sur le "ratage" en discours oral », L'information grammaticale, $\mathrm{n}^{\circ}$ 77, mars 1998, p. 43-47.
} 
l'intériorité subjective. Dans le cadre de ce travail, on laissera de côté le problème du bavard et de la voix infinie, ainsi que celui des déviances propres au dialogue bouffonnant, pour se consacrer particulièrement à l'indistinction des voix énonciatives. On sait que, dans La Route des Flandres en particulier, l'adresse de la voix est problématique, et qu'on ne sait plus très bien, au terme du roman, à qui Georges raconte son histoire. Parfois même, on ne sait même plus qui parle, comme le soulignent d'étranges discours attributifs hésitants :

[...] et Blum (ou Georges) : "C'est fini ?», et Georges (ou Blum) : «Je pourrais continuer», et Blum (ou Georges) : «Alors continue » $[\ldots](R F, 322)$

Or, cette interchangeabilité de la voix est intégrée à la logique même du dialogue. L'alternance des répliques dans l'interaction verbale se brouille, en particulier dans cet extrait de La Route des Flandres:

[B] je n'y comprends rien

[G] alors $\mathrm{m}$ es encore plus bête que Wack Je parie qu'il y a longtemps qu'il a compris

[B] plus bête que Wack très bien [...]

[G] ce type du bistrot n'avait pas trop l'air non plus de faire de différence entre sa femme et son chien

[B] peut-être que c'est un chien changé en femme

[G] peut-être

[B] savent jeter des sorts Secrets qui se perdent dommage Commode pourtant

[G] (B) alors il a transformé sa chèvre en fille ou sa sœur en chèvre et Vulcain je veux dire ce boiteux épousa la chèvrepied et ce bouc de frère venait la saillir dans sa maison c'est bien ça?

[B] (G) c'est ce qu'il a dit

[G] (B) alors c'était du lait de chèvre ?

[B] $(\mathrm{G})$ qui ?

[G] (B) celle qui était dans l'écurie ce matin celle qui se cache derrière ce paon mythologique celle dont la vue t'a plongé dans ce délire emmerdant poétique et coûteux [...] » $(R F, 280-281$.

Les lettres entre parenthèses et crochets sont de nous). 
Il s'agit d'un dilogue, dialogue à deux voix dans lequel l'alternance, en principe, est systématique, ce qui rend superflue l'attribution explicite du discours. Comme c'est forcément Blum qui parle en premier - c'est bien lui qui ne comprend rien à l'intrigue paysanne - la suite du dilogue ne devrait pas poser problème. Mais l'attribution normale des répliques, que nous avons figurée entre crochets, se heurte rapidement à une incohérence : la dernière réplique ne peut être prononcée que par Blum, puisque c'est Georges qui s'est plongé le matin dans ce "délire emmerdant». On est donc contraints de supposer que sans aucune indication, Blum a prononcé deux répliques à la suite, à un moment donné. On peut alors proposer, mais sans aucune certitude, une nouvelle alternance, qui figure entre parenthèses. Toute une zone de répliques demeure toutefois indécidable. Le lecteur, emporté par le flot bouffonnant, n'a pas claire conscience du brouillage, mais le texte crée pourtant à réception l'impression d'une voix non individualisée, qui participe pleinement de la situation d'énonciation. Car rappelons qu'à ce moment, Georges et Blum sont dans le noir et ne sont plus que deux « voix sans visage », que le monde s'en va en morceaux indistincts sous la pluie, et que l'on parle ici d'inceste, c'est-à-dire justement de la violation de la différenciation dans les choix sexuels (Blum dit bien que les paysans ne font pas la différence entre leur femme et leur sœur, ou entre leur femme et leur chien). C'est bien cette indifférenciation généralisée du monde et des perceptions qui guette les locuteurs et leurs voix dans le dialogue. Le principe de différence se perd entre les voix des divers personnages, et entre celles-ci et la voix narrative.

Au terme de cette étude, on avancera que le dialogue fonctionne toujours à double sens chez Simon, ce qui justifie aussi ses évolutions non linéaires, ses traitements divers et ses régulières disparitions. D'une part, le dialogue doit être le lieu de l'inscription d'une voix vive, qui viendrait animer le texte ; l'imitation soigneuse de style parlé, ainsi que l'invasion de certaines caractéristiques orales dans la narration, témoigne de ce désir nostalgique du texte écrit pour une voix présente et vive. Le texte tente d'inclure la voix sous une forme homogène, de l'introduire en son cœur même en gommant la frontière, comme en témoignent entre autres les formes mixtes de dis- 
cours rapporté et l'allégement de la ponctuation démarcative. Mais, ce faisant, il atténue aussi la spécificité de la voix narrative, ainsi que la différenciation subjective de chaque personnage.

La tentative forcément déceptive d'être fidèle à la voix, fantasme qui habite nous semble-t-il l'écriture simonienne, cohabite sans cesse avec la prise en compte de cette impossibilité, et laisse donc apparâ̂tre une voix impersonnelle, atopique et atemporelle, exténuée : une voix " décharnée » pour reprendre un mot de La Route des Flandres.

L'étude de la caractérisation de la lexie « voix » permet de poursuivre la réflexion dans le même sens : les descriptions laissent cohabiter le rêve de l'origine subjective orale avec l'étiolement de la voix - fidèles en cela à l'effet double, particulier, du texte simonien sur son lecteur : à la fois d'évidence et de retrait, de densité et d'étiolement, simultanément. 